PROCEEDINGS OF THE

AMERICAN MATHEMATICAL SOCIETY

Volume 125, Number 12, December 1997, Pages 3633-3639

S 0002-9939(97)04138-5

\title{
REPRESENTATIONS OF INVERSE FUNCTIONS
}

\author{
SABUROU SAITOH \\ (Communicated by Theodore W. Gamelin)
}

\begin{abstract}
Let $\phi: \hat{E} \rightarrow E$ be an arbitrary map from an abstract set $\hat{E}$ into an abstract set $E$. We shall use the theory of reproducing kernels to provide a general method for representing the inverse map $\phi^{-1}$ in terms of $\phi$. We give several concrete examples of this method.
\end{abstract}

\section{InTRODUCTION}

We shall consider an arbitrary mapping $p=\phi(\hat{p})$

$$
\phi: \hat{E} \longrightarrow E
$$

from an abstract set $\hat{E}$ into an abstract set $E$. Then, we shall consider the formal problem of representing the inverse map $\phi^{-1}$ in terms of $\phi$. Of course, the inverse is, in general, multi-valued. By using the theory of reproducing kernels ([1]), we shall first attack this general problem, and then, we shall establish a general principle to solve this problem in some general and reasonable settings. We shall also give several concrete examples.

\section{A GENERAL APPROACH}

Let $K(p, q)$ be a positive matrix on $E$ in the sense of Aronszajn-Moore, so that for any finite number of points. $\left\{p_{j}\right\}$ of $E$ and for any complex numbers $\left\{c_{j}\right\}$,

$$
\sum_{k} \sum_{j} \overline{c_{k}} c_{j} K\left(p_{k}, p_{j}\right) \geqq 0 .
$$

Then, there exists a uniquely determined functional Hilbert space $H_{K}$ consisting of functions on $E$ and admitting the reproducing kernel $K(p, q)$ with the properties that

(i) $K(\cdot, q) \in H_{K}$ for any $q \in E$, and

(ii) $(f(\cdot), K(\cdot, q))_{H_{K}}=f(q)$, for any $q \in E$ and for any $f \in H_{K}$.

We shall assume that $K(p, q)$ is expressible in the form

$$
K(p, q)=(\mathbf{h}(q), \mathbf{h}(p))_{\mathcal{H}} \quad \text { on } \quad E \times E
$$

Received by the editors May 15, 1995 and, in revised form, July 15, 1996.

1991 Mathematics Subject Classification. Primary 30C40.

Key words and phrases. Inverse function, reproducing kernel, the Riemann mapping function, Bergman kernel, Szegö kernel, multi-valued function, harmonic mapping, increasing function.

(C)1997 American Mathematical Society 
in terms of a Hilbert space $\mathcal{H}$-valued function $\mathbf{h}(p)$ on $E$. (Cf. [4], [5].) We further assume that

$$
\{\mathbf{h}(p) ; p \in E\} \quad \text { is complete in } \mathcal{H} .
$$

Then, for the linear transform of $\mathcal{H}$

$$
f(p)=(\mathbf{f}, \mathbf{h}(p))_{\mathcal{H}}, \quad \mathbf{f} \in \mathcal{H},
$$

the images $f(p)$ form precisely the Hilbert space $H_{K}$, and furthermore we have the isometrical mapping

$$
\|f\|_{H_{K}}=\|\mathbf{f}\|_{\mathcal{H}} .
$$

If the assumption (2.3) is not valid, then we have in (2.5), in general, the inequality

$$
\|f\|_{H_{K}} \leqq\|\mathbf{f}\|_{\mathcal{H}}
$$

Now, by using the mapping $\phi$ we define the function

$$
K_{\phi}(\hat{p}, \hat{q})=K(\phi(\hat{p}), \phi(\hat{q}))=(\mathbf{h}(\phi(\hat{q})), \mathbf{h}(\phi(\hat{p})))_{\mathcal{H}}
$$

on $\hat{E} \times \hat{E}$ and the linear mapping of $\mathcal{H}$

$$
f(\phi(\hat{p}))=(\mathbf{f}, \mathbf{h}(\phi(\hat{p})))_{\mathcal{H}}, \quad \mathbf{f} \in \mathcal{H} .
$$

Of course, $K_{\phi}(\hat{p}, \hat{q})$ is a positive matrix on $\hat{E}$, and so there exists a uniquely determined functional Hilbert space $H_{K_{\phi}}$ admitting the reproducing kernel $K_{\phi}(\hat{p}, \hat{q})$. Then, we obtain, from (2.5) and (2.6)

Theorem 2.1. For an arbitrary mapping $\phi$ in (1.1), the functions $f(\phi(\hat{p}))$ in (2.8) form the reproducing kernel Hilbert space $H_{K_{\phi}}$ and we have the inequality

$$
\|f(\phi)\|_{H_{K_{\phi}}} \leqq\|f\|_{H_{K}} .
$$

Isometry holds here if and only if

$$
\{\mathbf{h}(p) ; p \in \text { Range } \phi\} \quad \text { is complete in } \mathcal{H} .
$$

Furthermore, we have

Theorem 2.2. Let $\phi: \hat{E} \longrightarrow E$ be arbitrary and let $\hat{f} \in H_{K_{\phi}}$. If $f^{*} \in H_{K}$ satisfies

$$
\hat{f}=f^{*}(\phi) \quad \text { and } \quad\|\hat{f}\|_{H_{K_{\phi}}}=\left\|f^{*}\right\|_{H_{K}},
$$

then

$$
f^{*}(p)=(\hat{f}(\cdot), K(\phi(\cdot), p))_{H_{K_{\phi}}} .
$$

Proof. Let $N(\phi)$ denote the set of $\mathbf{f} \in \mathcal{H}$ such that

$$
(\mathbf{f}, \mathbf{h}(\phi(\hat{p})))_{\mathcal{H}}=0 \quad \text { on } \quad \hat{E},
$$

and let $[N(\phi)]^{\perp}$ denote its orthogonal complement in $\mathcal{H}$. Then, in the mapping (2.8) we can take $\mathbf{f}^{*} \in[N(\phi)]^{\perp}$ satisfying

$$
\hat{f}(\hat{p})=\left(\mathbf{f}^{*}, \mathbf{h}(\phi(\hat{p}))\right)_{\mathcal{H}}
$$

and

$$
\|\hat{f}\|_{H_{K_{\phi}}}=\left\|\mathbf{f}^{*}\right\|_{\mathcal{H}}
$$


Then, for

$$
f^{*}(p)=\left(\mathbf{f}^{*}, \mathbf{h}(p)\right)_{\mathcal{H}},
$$

we have

$$
\hat{f}(\hat{p})=f^{*}(\phi(\hat{p}))
$$

satisfying (2.11).

Then, for the orthogonal projection $P$ from $\mathcal{H}$ onto $[N(\phi)]^{\perp}$ we have the desired result

$$
\begin{aligned}
f^{*}(p) & =\left(\mathbf{f}^{*}, \mathbf{h}(p)\right)_{\mathcal{H}} \\
& =\left(P_{[N(\phi)]^{\perp}} \mathbf{f}^{*}, \mathbf{h}(p)\right)_{\mathcal{H}} \\
& =\left(\mathbf{f}^{*}, P_{[N(\phi)]^{\perp}} \mathbf{h}(p)\right)_{\mathcal{H}} \\
& =(\hat{f}(\cdot), K(\phi(\cdot), p))_{H_{K_{\phi}}} .
\end{aligned}
$$

Now, in Theorem 2.2, we shall assume that the mapping $\phi$ is onto $\hat{E}$ and so isometry between $H_{K}$ and $H_{K_{\phi}}$.

If we know the mapping $p=\phi(\hat{p})$ from $\hat{E}$ to $E$, then the isometrical mapping from $H_{K}$ onto $H_{K_{\phi}}$ is given by

$$
f(p) \in H_{K} \longrightarrow f(\phi(\hat{p})) \in H_{K_{\phi}} .
$$

If we know the (in general, multi-valued) inverse $\hat{p}=\phi^{-1}(p)$ of $p=\phi(\hat{p})$, then for $\hat{f} \in H_{K_{\phi}}$, the function $\hat{f}\left(\phi^{-1}(p)\right)$ is a single-valued function on $E$ and the isometrical mapping from $H_{K_{\phi}}$ onto $H_{K}$ is given by

$$
\hat{f} \in H_{K_{\phi}} \longrightarrow \hat{f}\left(\phi^{-1}(p)\right) \in H_{K} .
$$

In general, Theorem 2.2 establishes the isometrical mapping

$$
\hat{f} \in H_{K_{\phi}} \longrightarrow f^{*} \in H_{K},
$$

explicitly in terms of the reproducing kernel $K(p, q)$ on $E$, the mapping $\phi$ and the reproducing kernel Hilbert space $H_{K_{\phi}}$. This fact means that Theorem 2.2 gives, in a sense, a method for constructing the inverse $\phi^{-1}$.

Indeed, for any point $p \in E$ and for any fixed function $\hat{f} \in H_{K_{\phi}}$ we first construct the function $f^{*}$ by $(2.12)$. Then, we have the inclusion relationship

$$
\phi^{-1}(p) \subset\left\{\hat{p} \in \hat{E} ; \hat{f}(\hat{p})=f^{*}(p)\right\} .
$$

Hence, we will be able to look for all the inverses $\phi^{-1}(p)$ in the point set in the righthand side in (2.20), by using a suitable Hilbert space $H_{K}$ and a suitable function $\hat{f} \in H_{K_{\phi}}$.

\section{ReAsonable SETtingS}

We shall analyze the principle in Section 2 to represent the inverse $\phi^{-1}$ in (1.1) interms of $\phi$. 
In order to use the formula (2.12) we need

(I) a concrete structure for the Hilbert space $H_{K_{\phi}}$, admitting its reproducing kernel $K_{\phi}(\hat{p}, \hat{q})$ defined by $(2.7)$

and

(II) for a function $\hat{f}$ belonging to $H_{K_{\phi}}$, its inverse $\hat{f}^{-1}$.

Then we have the inverse function of $\phi$

$$
\hat{p}=\hat{f}^{-1}\left(f^{*}(p)\right),
$$

in $(2.20)$.

For (I), if the Hilbert space $H_{K}$ admitting the reproducing kernel $K(p, q)$ on $E$ is concretely given, then the transformed Hilbert space $H_{K_{\phi}}$ will be constructed by a unified method stated in Theorem 2.1 and Theorem $2.2([6])$.

If the inner product in $H_{K}$ is given by an integral form, then the inner product in $H_{K_{\phi}}$ will be given by some integral form induced by the mapping $\phi$, as we shall see in examples.

For (II), if the identity mapping belongs to $H_{K_{\phi}}$, then our situation will become, of course, extremely simple.

\section{EXAMPLES}

Following our general principle, we shall give typical examples. These examples will show that concrete reproducing kernels have great value from also the viewpoint of representations of inverse functions.

4.1. The Riemann mapping function. Let $\triangle$ be the unit disc $\{|z|<1\}$ and $D$ an arbitrary bounded (for simplicity) domain on the $Z$-plane $(Z=X+i Y)$. Let $z=\varphi(Z)$ be a Riemann mapping function from $D$ onto $\triangle$, which is analytic and univalent on $D$. Let $K_{\triangle}(z, \bar{u})$ be the Bergman kernel

$$
K_{\triangle}(z, \bar{u})=\frac{1}{\pi(1-\bar{u} z)^{2}}
$$

on $\triangle$ for the Bergman space $H_{K_{\triangle}}$ comprised of all analytic functions $f(z)$ on $\triangle$ with finite norms

$$
\left\{\iint_{\triangle}|f(z)|^{2} d x d y\right\}^{\frac{1}{2}}<\infty \quad(z=x+i y) .
$$

Then, we can see directly that

$$
K_{\triangle, \varphi}(\varphi(Z), \overline{\varphi(U)})=\frac{1}{\pi(1-\overline{\varphi(U)} \varphi(Z))^{2}}
$$

is the reproducing kernel for the Hilbert space $H_{K_{\triangle, \varphi}}$ comprised of all analytic functions $\hat{f}(Z)=f(\varphi(Z))\left(f \in H_{K_{\Delta}}\right)$ with finite norms

$$
\left\{\iint_{D}|\hat{f}(Z)|^{2}\left|\varphi^{\prime}(Z)\right|^{2} d X d Y\right\}^{\frac{1}{2}}<\infty .
$$

Hence, we have the inverse formula by (2.12) and by using the identity for $\hat{f}$ in $(2.20)$

$$
\varphi^{-1}(z)=\frac{1}{\pi} \iint_{D} \frac{Z\left|\varphi^{\prime}(Z)\right|^{2}}{(1-z \overline{\varphi(Z)})^{2}} d X d Y .
$$


When $Z=\varphi^{-1}(z)$ is not one-to-one, if we consider $D$ as a Riemann surface $\varphi^{-1}(\triangle)$ spread over $\mathbb{C}$, counting multiplicity, then the formula (4.4) is still valid. By this method, we can, in general, overcome the multi-valuedness of the inverse functions.

When $D$ is a bounded domain whose boundary $\partial D$ is an analytic Jordan curve, we recall the Szegö reproducing kernel

$$
\frac{1}{2 \pi(1-\bar{u} z)}
$$

for the Hilbert space consisting of all analytic functions $f(z)$ on $\triangle$ such that $|f(z)|^{2}$ has a harmonic majorant and with finite norm

$$
\left\{\int_{\partial \Delta}|f(z)|^{2}|d z|\right\}^{\frac{1}{2}}<\infty .
$$

Here, $f(z)$ means the Fatou nontangential boundary value on $\partial \Delta$. Then, we obtain similarly the simple inverse formula

$$
\varphi^{-1}(z)=\frac{1}{2 \pi} \int_{\partial D} \frac{Z\left|\varphi^{\prime}(Z)\right|}{1-z \overline{\varphi(Z)}}|d Z| .
$$

The very elementary formulas (4.4) and (4.7) can be derived directly and easily, but it seems that they cannot be found in any articles.

Note that the Riemann mapping function $\varphi(Z)$ satisfying $\varphi\left(Z_{0}\right)=0 \quad\left(Z_{0} \in D\right)$ is expressible in the form

$$
\varphi(Z)=\sqrt{\frac{\pi}{K_{D}\left(Z_{0}, \bar{Z}_{0}\right)}} \int_{Z_{0}}^{Z} K_{D}\left(\zeta, \overline{Z_{0}}\right) d \zeta
$$

in terms of the Bergman kernel $K_{D}(\zeta, \bar{Z})$ on the domain $D$ (see, for example, [3]).

4.2. Harmonic mappings. Note that on $\triangle$

$$
\frac{1-|\bar{u}|^{2}|z|^{2}|2-\bar{u} z|^{2}}{|1-\bar{u} z|^{4}}
$$

and

$$
\frac{1-|\bar{u}|^{2}|z|^{2}}{|1-\bar{u} z|^{2}}
$$

are the reproducing kernels for the Hilbert spaces consisting of all harmonic functions $u(z)$ on $\triangle$ with finite norm

$$
\left\{\frac{1}{\pi} \iint_{\triangle}|u(z)|^{2} d x d y\right\}^{\frac{1}{2}}<\infty
$$

and

$$
\left\{\frac{1}{2 \pi} \int_{\partial \triangle}|u(z)|^{2}|d z|\right\}^{\frac{1}{2}}<\infty,
$$

respectively. For the latter case we need the assumption that $|u(z)|^{2}$ has a harmonic majorant on $\triangle$ and $u(z)(z \in \partial \triangle)$ means the Fatou nontangential boundary value as in the Szegö case (see, for example, [2] and [5]). As in the Riemann mapping function we can obtain the representations of the inverse functions for harmonic mappings of $D$ onto $\triangle$. 
4.3. Increasing functions. Note first that

$$
\min (x, y) \quad(x, y>0)
$$

is the reproducing kernel for the Hilbert space $H(0, a)(0<a \leqq \infty)$ consisting of all real-valued functions $f(x)$ on $[0, a)$ such that $f(x)$ are absolutely continuous on $[0, a), f(0)=0$ and with finite norms

$$
\left\{\int_{0}^{a} f^{\prime}(x)^{2} d x\right\}^{\frac{1}{2}}<\infty
$$

Similarly, for an increasing function $x=\varphi(\hat{x})$ from $[0, b)(0<b \leqq \infty)$ onto $[0, a)$ of $C^{1}$-class satisfying

$$
\varphi^{\prime}(\hat{x})>0 \quad \text { on } \quad[0, b)
$$

the function

$$
\min (\varphi(\hat{x}), \varphi(\hat{y})) \quad \text { on } \quad[0, b) \times[0, b)
$$

is the reproducing kernel for the Hilbert space $H_{\varphi}$ consisting of all functions $\hat{f}(\hat{x})=$ $f(\varphi(\hat{x}))(f \in H(0, a))$, such that $\hat{f}(\hat{x})$ are absolutely continuous on $[0, b), \hat{f}(0)=0$ and with finite norms

$$
\left\{\int_{0}^{b} \hat{f}^{\prime}(\xi)^{2} \frac{d \xi}{\varphi^{\prime}(\xi)}\right\}^{\frac{1}{2}}<\infty,
$$

as we can see directly. Hence, by (2.12) and by using the identity as $\hat{f}$ in (2.20), we have the formula

$$
\begin{aligned}
\varphi^{-1}(x) & =\int_{0}^{b}\{\min (\varphi(\xi), x)\}^{\prime} \frac{d \xi}{\varphi^{\prime}(\xi)} \\
& =\int_{0}^{b}\left(\frac{2}{\pi} \int_{0}^{\infty} \frac{\sin (\varphi(\xi) t) \sin x t}{t^{2}} d t\right)^{\prime} \frac{d \xi}{\varphi^{\prime}(\xi)} \\
& =\frac{2}{\pi} \int_{0}^{b} \int_{0}^{\infty} \frac{\cos (\varphi(\xi) t) \sin x t}{t} d t d \xi .
\end{aligned}
$$

In particular, we have

$$
\sqrt[n]{x}=\frac{2}{\pi} \int_{0}^{\infty} \int_{0}^{\infty} \frac{\cos \left(\xi^{n} t\right) \sin x t}{t} d t d \xi
$$

\section{ACKNOWLEDGMENT}

This research was partially supported by the Japanese Ministry of Education, Science and Culture; Grant-in-Aid Scientific Research, General Research (C) 06640201 .

\section{REFERENCES}

1. N. Aronszajn, Theory of reproducing kernels, Trans. Amer. Math. Soc. 68 (1950), 337-404. MR 14:479c

2. __ Green's functions and reproducing kernels, Proceedings of the Symposium on Spectral Theory and Differential Problems, Oklahoma A. and M. College, Oklahoma, (1951), pp. 355411. MR 15:878a

3. S. Bergman, The kernel function and conformal mapping, Amer. Math. Soc. Providence, R.I., (1970). MR 58:22502 
4. S. Saitoh, Hilbert spaces induced by Hilbert space valued functions, Proc. Amer. Math. Soc. 89 (1983), 74-78. MR 84h:44001

5. - Theory of reproducing kernels and its applications, Pitman Res. Notes in Math. Series 189, Longman Scientific \& Technical, England, (1988). MR 90f:46045

6. __ Decreasing principles in transforms of reproducing kernel Hilbert spaces, Mathematica Montisnigri 1 (1993), 99-109. MR 94i:46042

Department of Mathematics, Faculty of Engineering, Gunma University, Kiryu 376, JAPAN

E-mail address: ssaitoh@eg.gunma-u.ac.jp 